Check for updates

Cite this: RSC Adv., 2017, 7, 48429

Received 23rd August 2017 Accepted 8th October 2017

DOI: 10.1039/c7ra09338a

rsc.li/rsc-advances

\section{One-pot hydrothermal synthesis of luminescent silicon-based nanoparticles for highly specific detection of oxytetracycline via ratiometric fluorescent strategy $\dagger$}

\begin{abstract}
Na Xu, (D) a Yaqing Yuan, ${ }^{a}$ Jian-Hang Yin, ${ }^{a}$ Xue Wang ${ }^{a}$ and Lei Meng (D) *ab
We report a one-pot hydrothermal synthesis of water dispersible luminescent silicon-based nanoparticles (SiNPs) functionalized by amino groups, starting from aminopropyltriethoxy silane and ascorbic acid. The structure, surface chemistry and optical features of the resulting SiNPs are well characterized by transmission electron microscopy, X-ray photoelectron spectroscopy, X-ray diffraction, Fourier transform infrared spectroscopy, UV-vis absorption spectra, transient and steady-state fluorescence. The products show high tolerability to extreme $\mathrm{pH}$ and high ionic strengths, and excellent photo-stability under UV irradiation, laying the foundation for their practical applications. As a fluorescent sensor, SiNPs have been used to sensitively determine oxytetracycline (OTC); particularly, they can distinguish OTC from other antibiotics with similar structure among the tetracyclines' (TCs') family. In the presence of OTC, the blue emission at $436 \mathrm{~nm}$ of SiNPs is dramatically quenched. Meanwhile, a new green emission, peaking at $502 \mathrm{~nm}$, appears and increases with the titration of OTC. Thereby, the trace of OTC can be monitored by SiNPs based on ratio between the two fluorescent intensities $\left(I_{502} / I_{436}\right)$. The $I_{502} / I_{436}$ displays a good linear response to OTC concentrations in the range of $0.2 \mu \mathrm{M}$ to $20 \mu \mathrm{M}$ with a detection limit of $0.18 \mu \mathrm{M}$. Finally, this newly developed protocol is successfully used to determine OTC in milk and the observed recoveries suggest the sensor can serve as an effective tool to detect OTC in foodstuffs.
\end{abstract}

\section{Introduction}

SiNPs, consisting of crystal silicon and its oxide, show highly luminescent properties and have received much attention in many fields such as solar energy, ${ }^{\mathbf{1 , 2}}$ photonic circuits ${ }^{3}$ and fluorescent sensors. ${ }^{4}$ During the past years, numerous approaches to prepare luminescent SiNPs have been developed. ${ }^{5-11}$ But most reported SiNPs are prone to oxidation in the ambient environment and are not easily dispersed in common solvents. Consequently, further surface modification should be employed to improve their stability and solubility. As one of aqueous synthetic strategies, hydrothermal synthesis ${ }^{12,13}$ can directly fabricate highly fluorescent and photostable SiNPs in an aqueous phase. This rapid, facile and efficient approach has received increasing interests. Recently, our group has successfully prepared a water-dispersible luminescent SiNPs via hydrothermal method to detect manganous ions. ${ }^{\mathbf{1 4}}$ Unique optical properties combined with excellent biocompatibility

${ }^{a}$ College of Materials Science and Engineering, Jilin Institute of Chemical Technology, Jilin 132022, China. E-mail: ml_0512@163.com

${ }^{b}$ College of Science, Jilin Institute of Chemical Technology, Jilin 132022, China

$\dagger$ Electronic supplementary information (ESI) available. See DOI: 10.1039/c7ra09338a offer SiNPs opportunities for design of high-performance fluorescent sensors. To date, we have witnessed vast advancements in the development of silicon nanomaterial-based optical analytical platforms for sensing biological species, such as metal ions, ${ }^{15,16}$ RNA and DNA, ${ }^{17,18}$ cell labeling, ${ }^{19,20}$ intracellular $\mathrm{pH},{ }^{21}$ and so on. However, the detection of various antibiotics using label-free SiNPs is very limited.

Oxytetracycline (OTC), containing four condensed aromatic rings, is a member of the broad-spectrum TCs' family of antibiotics. Other three derivatives, including tetracycline (TC), doxycycline (DC) and chlortetracycline (CTC), are structurally similar to OTC (Scheme S1 $\dagger$ ). Owing to its strong antibacterial activity against bacteria, OTC is widely used for the treatment or prevention of infections. It has become the most common growth promoter for livestock and aquaculture due its low cost and high efficiency. However, widespread use has led to the presence of OTC residues in animal foods, such as meat, milk and eggs/chicken, which is a significant problem in terms of food safety. ${ }^{22,23}$ For the purpose of detecting TCs, diverse techniques have been developed including high performance liquid chromatography (HPLC), ${ }^{24}$ liquid chromatography-mass spectrometry (LC/MS), ${ }^{25}$ enzyme-linked immunoassay (ELISA) ${ }^{26}$ and electrochemical analysis. ${ }^{27}$ Nevertheless, these traditional methods need complicated instruments and operation, or 


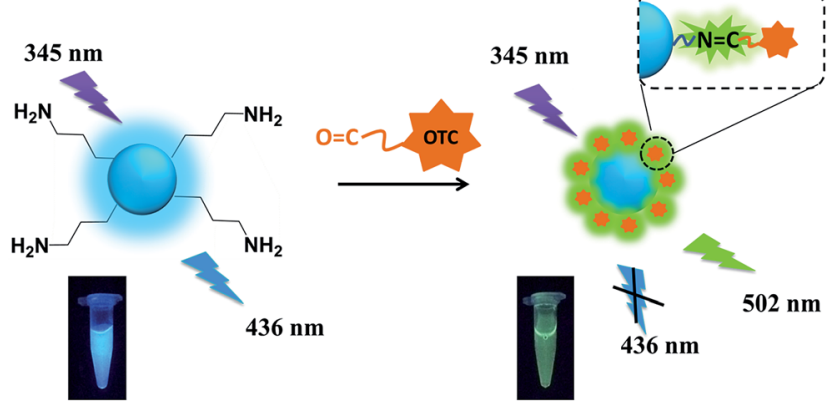

Scheme 1 Schematic illustration of detection of OTC using SiNPs via radiometric fluorescence strategy.

require sample pretreatment. Thus, facile, fast and sensitive method for detecting OTC residues in foodstuffs is urgently needed.

By virtue of its high sensitivity and nondestructive nature,,$^{28,29}$ fluorescent sensor is more suitable to detect OTC in drugs or human food than other platforms. So far, however, most of OTC-based sensors reply on the changes of single emission intensity during the detection. ${ }^{30-34}$ The interferences from other environmental parameters (e.g. $\mathrm{pH}$, temperature, ionic strength) may affect the emission intensity and thus the sensitivity of sensor. On the other hand, specific detection of OTC among TCs' family is very poor due to their similar structure and chemical properties (Scheme S1†).

Herein, we develop a novel fluorescent sensor for OTC based on SiNPs, prepared by one-pot hydrothermal method. Comparing to other traditional sensors, it can determine OTC through ratiometric fluorescent strategy. The resulting SiNPs are functionalized by amino groups, and OTC has amounts of hydroxyl and carbonyl groups. Owing to the electrostatic forces and nucleophilic addition reaction, the SiNPs can easily combine to OTC and finally form SiNPs-OTC complex with $\mathrm{C}=\mathrm{N}$ binding Schiff base, which possesses green emission at $502 \mathrm{~nm}$ (Scheme 1). Consequently, SiNPs-OTC mixture exhibit dual-emission fluorescent property. The blue fluorescent emission of SiNPs is quenched by OTC based on inner filter effect (IFE), while the green one from Schiff base increases linearly and acts as the reference signal. In addition, this fluorescent sensor can specifically recognize OTC among complicated TCs' family.

\section{Experimental}

\subsection{Chemicals and materials}

(3-Aminopropyl) triethoxysilane (APTES), ascorbic acid (AA), OTC and its three analogues (TC, CTC and DC), trichloroacetic acid and other routine chemicals were obtained from Tianjin Guangfu Fine Chemical Research Institute. Britton-Robinson (BR) buffer solutions are employed to study the stability of SiNPs toward different $\mathrm{pH}$ values, which were prepared by mixing acid solutions containing $0.04 \mathrm{M}$ boric acid, $0.04 \mathrm{M}$ acetate, and $0.04 \mathrm{M}$ phosphate, using $0.20 \mathrm{M} \mathrm{NaOH}$ solution to adjust $\mathrm{pH}$ value. All reagents were of analytical reagent grade and were used without further purification. Ultrapure water obtained from a Milli-Q ultrapure (18.2 $\left.\mathrm{M} \Omega \mathrm{cm}^{-1}\right)$ system was used in all experiments.

\subsection{Apparatus}

UV-vis absorption instrument (Lambda 950UV/Vis/NIR, PerkinElmer, USA) was used to measure the optical absorption spectra. The fluorescence spectra (LS-55, PerkinElmer, USA) were tested at an excitation wavelength of $345 \mathrm{~nm}$. Transmission electron microscopy (TEM) images are collected by a Tecnai G2 S-Twin F20 transmission electron microscope (FEI, USA). X-ray photoelectron spectroscopy (XPS) measurements are obtained by using an ESCALAB 250 spectrometer with a monochromic X-ray source ( $\mathrm{Al} \mathrm{K} \alpha$ line, $1486.6 \mathrm{eV}$ ). Crystal structure was measured by a Rigaku D/Max-RA X-ray diffractometer (XRD) with $\mathrm{Cu} \mathrm{K} \alpha$ radiation $(\lambda=1.5406 \AA)$. Fourier transform infrared (FT-IR) spectra were collected with a Spectrum Two spectrometer (PerkinElmer, USA). The thermal behavior was studied by a Pyris 1 thermogravimetric analyzer under nitrogen at heating rate of $10{ }^{\circ} \mathrm{C} \mathrm{min}^{-1}$ (PerkinElmer, USA). The measurement of time decay of fluorescence intensity is carried out on a FLS920 Edinburgh Instruments (U.K.), being combined with fluorescence lifetime and steady state spectrometer. Dynamic light scattering (DLS) and zeta potential measurements were performed using a nano ZS90 laser scattering particles size and zeta-potential analyzer (Malvern, U.K.). The $\mathrm{pH}$ values were measured with FE 20 pH meter (Mettler-Toledo, China).

\subsection{Preparation of luminescent SiNPs}

SiNPs with the maximum emission at $436 \mathrm{~nm}$ exited by $345 \mathrm{~nm}$ were fabricated by a simple one-step hydrothermal route. Briefly, $3 \mathrm{~mL}$ APTES and $30 \mu \mathrm{L}$ AA $(100 \mathrm{mM})$ were dissolved in $12 \mathrm{~mL}$ ultrapure water. After stirring for $10 \mathrm{~min}$, the mixture was transferred into a Teflon-lined stainless-steel autoclave of $50 \mathrm{~mL}$ and heated to $240{ }^{\circ} \mathrm{C}$ for $3 \mathrm{~h}$. The obtained solution was filtered extensively with $0.22 \mu \mathrm{M}$ ultrafiltration membrane to remove the large particles, and then dialyzed extensively with a dialysis membrane (3500 Da) in ultrapure water for $12 \mathrm{~h}$.

\subsection{Detection of OTC}

The OTC detection procedure was carried out in aqueous solution. OTC was added into $2 \mathrm{~mL}$ of $4 \mathrm{mM}$ probe solution one by one with the final concentrations of 0.2 to $30 \mu \mathrm{M}$. After incubation for $2 \mathrm{~min}$, the corresponding fluorescence spectra of the solutions were then recorded under excitation at $345 \mathrm{~nm}$. The ratio changes of the fluorescence intensities $\left(I_{502} / I_{436}\right)$ were used to construct the curves and evaluate the performance of SiNPs toward OTC. The selectivity of this sensing system for OTC activity was assessed by using other TCs' family and the following substances as $\mathrm{Na}^{+}, \mathrm{K}^{+}, \mathrm{Cl}^{-}, \mathrm{CO}_{3}{ }^{2-}, \mathrm{HCO}_{3}{ }^{-}, \mathrm{PO}_{4}{ }^{3-}$, $\mathrm{HPO}_{4}{ }^{2-}$ and $\mathrm{H}_{2} \mathrm{PO}_{4}{ }^{-}$. All the measurements are performed at ambient conditions.

\subsection{Sample preparation of milk}

The milk was obtained from the local farm. The procedure described previously to precipitate the proteins and fat in milk 
was modified slightly in the present study. Briefly, $1 \mathrm{~mL}$ milk sample was diluted into $10 \mathrm{~mL}$ ultrapure water and subse-

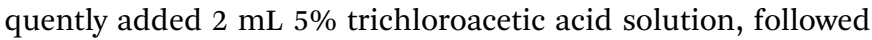
by vortexing for $1 \mathrm{~min}$ and ultrasonification for $30 \mathrm{~min}$. After that, the mixture was centrifuged at $10000 \mathrm{rpm}$ for $10 \mathrm{~min}$. Then the obtained supernatant was filtered extensively with $0.22 \mu \mathrm{M}$ ultrafiltration membrane to remove the precipitate. The filtrate was stored at $4{ }^{\circ} \mathrm{C}$ for further analyzing.

\section{Results and discussion}

\subsection{Preparation and optimization of SiNPs}

SiNPs are synthesized in aqueous solution via a one-pot hydrothermal method. The mixture, consisting of APTES and $\mathrm{AA}$, is put into a reaction still to be heated at a defined temperature. Consequently, an intense blue emission at $436 \mathrm{~nm}$ has been observed when existed at $345 \mathrm{~nm}$. Afterward, several experimental factors such as molar ratios of reactants, temperature and continuous reaction time, are respectively optimized to achieve SiNPs with better fluorescence properties. As shown in Fig. S1, $\dagger$ the SiNPs with highest fluorescence at $436 \mathrm{~nm}$ is obtained under suitable conditions, where the molar ratio of APTES to AA is chosen at 100:1 and temperature of reaction is fixed at $240{ }^{\circ} \mathrm{C}$ for $3 \mathrm{~h}$.

\subsection{Characterization of SiNPs}

Fig. 1a and its inset respectively display TEM image and DLS size distribution of SiNPs prepared basing on optimum conditions. The resulting SiNPs are spherical particles that appear to have high dispersity with diameter of about $4.5 \mathrm{~nm}$. XPS is used to analyze the valence state of elements, and surface chemical bonds of the products. As shown in Fig. $1 \mathrm{~b}$ and $\mathrm{S} 2, \dagger$ four characteristic peaks at 102.4, 284.7, 523.3 and $399.3 \mathrm{eV}$ represent respectively for $\mathrm{Si} 2 \mathrm{p}, \mathrm{C} 1 \mathrm{~s}, \mathrm{O} 1 \mathrm{~s}$ and N1s, suggesting the presence of $\mathrm{Si}^{4+}, \mathrm{C}, \mathrm{O}$ and $\mathrm{N}$ in SiNPs. There is almost no characteristic peak toward zero valence silicon $\left(\mathrm{Si}^{0}\right)$ observed in XPS measurement, suggesting Si-O bonds are mainly exists in the SiNPs. ${ }^{35,36}$ Furthermore, XRD pattern (Fig. 1c) of SiNPs represents one single diffraction peak at about $2 \theta=22^{\circ}$, assigned to the (101) planes of silicon oxide (ICDD 39-1426). Thereby, results from XPS and XRD single that SiNPs mainly consist of silicon oxide. In addition, FT-IR spectrum of SiNPs is shown in Fig. 1d and several distinct transmission peaks are represented in the range of $800-4000 \mathrm{~cm}^{-1}$. Particularly, there exist two characteristic peaks at around 3440 and $1580 \mathrm{~cm}^{-1}$; and they are corresponding to $\mathrm{N}-\mathrm{H}$ bond bending and the $\mathrm{N}-\mathrm{H}$ stretching vibration, respectively. ${ }^{12,37}$ One intense peak at around $1100 \mathrm{~cm}^{-1}$ can be indexed to the vibrational stretch of Si-O bonding. Moreover, zeta potential $(\zeta)$ of SiNPs in aqueous media ( $\mathrm{pH}<7.0$ ) is $2.58 \mathrm{mV}$. FT-IR and zeta potential analysis demonstrates the surface of resulting SiNPs possess amounts of amino groups with sufficient positive charges. As shown in Scheme $\mathrm{S} 2, \uparrow$ the APTES can be easily hydrolyzed in aqueous solution. Owning to AA processes amounts of hydroxyl groups, hydrolyzed products can polymerize with AA and then form particles with $-(\mathrm{Si}-\mathrm{O})_{x}$ structure, ${ }^{38,39}$ which is terminated by amine groups during high temperature hydrothermal process.

UV-vis absorption spectrum of transparent SiNPs contained solution is given in Fig. 2a, where it shows one single broad absorption band centered at $270 \mathrm{~nm}$. The highest emission (a)

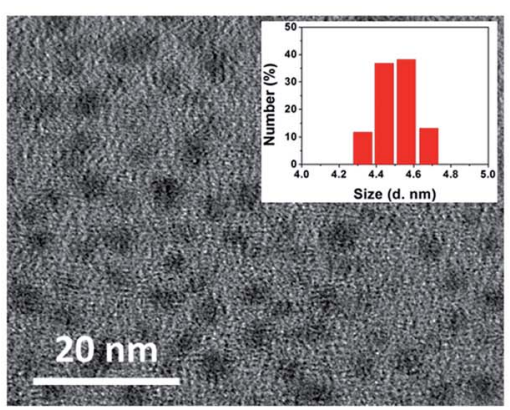

(c)

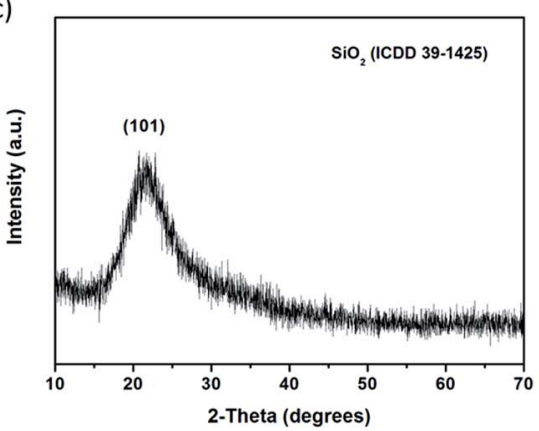

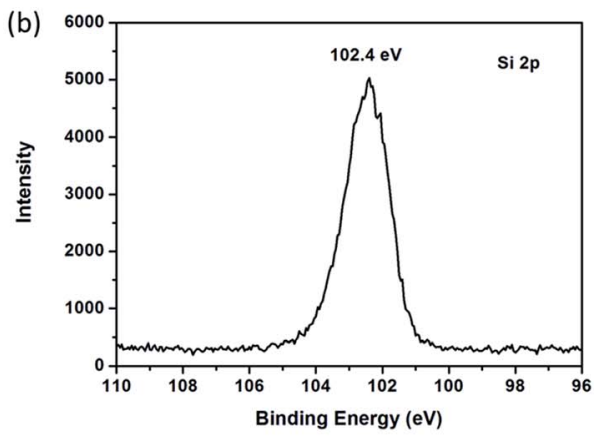

(d)

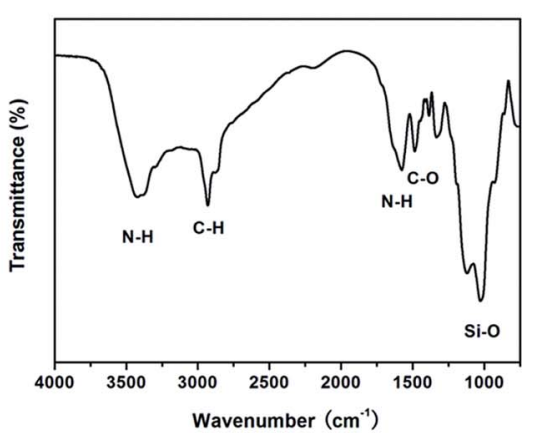

Fig. 1 (a)TEM image of SiNPs, inset shows size distribution of SiNPs by DLS measurement. (b) Characteristics toward Si2p in SiNPs by XPS. (c) XRD pattern and (d) FT-IR spectrum of SiNPs. 
(a)

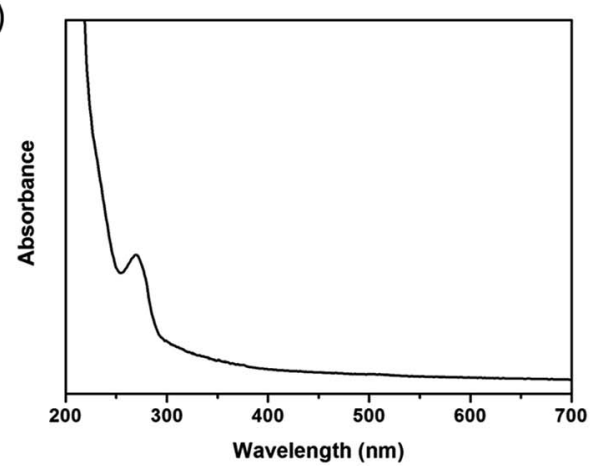

(c)

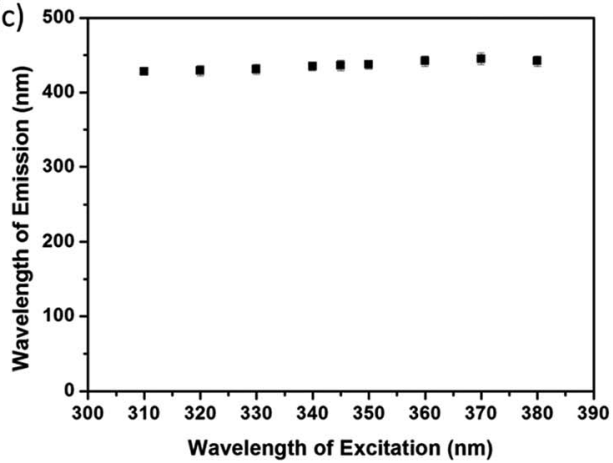

(b)

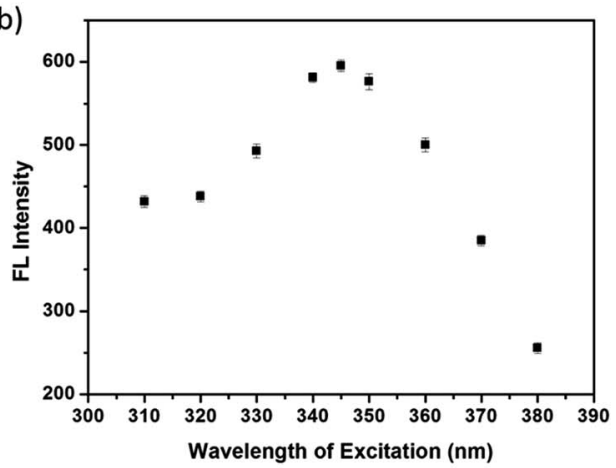

(d)

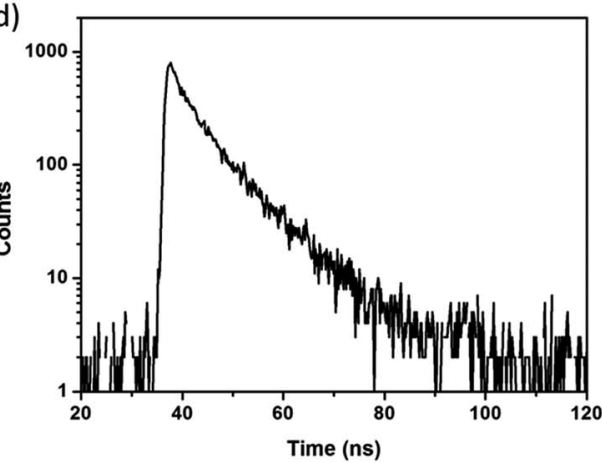

Fig. 2 (a) UV-vis absorbance of SiNPs. (b) Fluorescence intensities and (c) position of emission spectra of SiNPs when excited by different wavelengths. (d) Lifetime decay profile of SiNPs.

intensity at $436 \mathrm{~nm}$ is obtained upon exited by $345 \mathrm{~nm}$ (Fig. 2b). According to the previous literature, ${ }^{\mathbf{4 0}}$ this absorption band is due to some form of oxygen deficiency in the $\mathrm{SiO}_{2}$ network such as oxygen vacancy. Furthermore, oxygen vacancy and peroxy linkage $\equiv \mathrm{Si}-\mathrm{O}-\mathrm{O}-\mathrm{Si} \equiv$ can form self-trapped excitation in $\mathrm{SiO}_{2}{ }^{41}$ which lead to blue fluorescent emission through recombination (Fig. 2b).

Fig. 2c shows luminescent emission spectra of SiNPs when existed by various wavelengths in the range of $310-380 \mathrm{~nm}$. It is found that the emission bands of SiNPs are located in the narrow range of 428-445 nm, suggesting SiNPs exhibit well size distribution and/or low density of emissive traps at the surface. ${ }^{10,42}$ In addition, the fluorescence decay curve of SiNPs is tested (Fig. 2d), and the decay parameters as lifetime $(\tau)$, amplitude of component $(B)$ and fitting degree $\left(\chi^{2}\right)$ are listed in Table 1 . The result shows biexponentially decay consisting nanosecond (7.71 ns) and microsecond lifetimes (9.63 $\mu \mathrm{s})$. According to the ref. 43 and 44, the shorter lifetime arises from surface related recombination, which is easily influence by size of SiNPs and its surface chemistry. However, the longer lifetime is attributed to recombination in SiNPs.

Table 1 Lifetime parameters measured by fluorescence decay of SiNPs $\left(\lambda_{\mathrm{em}}=436 \mathrm{~nm}\right)$ and SiNPs-OTC complex $\left(\lambda_{\mathrm{em}}=502 \mathrm{~nm}\right)$ under the excitation of $365 \mathrm{~nm}$

\begin{tabular}{llrlrl}
\hline Samples & $\tau_{1}(\mathrm{~ns})$ & $\tau_{2}(\mu \mathrm{s})$ & \multicolumn{1}{c}{$B_{1}$} & \multicolumn{1}{c}{$B_{2}$} & \multicolumn{1}{c}{$\chi^{2}$} \\
\hline SiNPs & 7.71 & 9.63 & 598.4 & 90.06 & 1.270 \\
SiNPs-OTC & 7.19 & 12.36 & 584.89 & 132.18 & 1.268
\end{tabular}

\subsection{Stability of SiNPs}

To further evaluate the stability of SiNPs, their fluorescence intensities are recorded under various extreme conditions, including $\mathrm{pH}$, ionic strengths and irradiation. Fig. S3a $\uparrow$ shows that the fluorescence intensity of the SiNPs at $436 \mathrm{~nm}$ does not change much in responding to $\mathrm{pH}$ variation from 2.0 to 12.0. Such a good resistance to extreme acid and base is essentially attributed to the high adjusting ability of amino groups in SiNPs, which are easily to be protonated or deprotonated depending on conditions as reported. In addition, similar dull response is also observed for SiNPs in the presence of different amount of $\mathrm{NaCl}$ (Fig. S3b $\dagger$ ). It is excited to discover no much effect of dense $\mathrm{NaCl}$ on SiNPs fluorescence, lay the foundation to apply this unique material to biological system with high ionic strength. Moreover, the fluorescence intensity of SiNPs can be kept stable for more than $100 \mathrm{~min}$ under exposure to strong light of $365 \mathrm{~nm}$ (Fig. S3c $\dagger$ ), illustrating the good photostability of this material. Therefore, the high stability of the as-prepared SiNPs endows it with the ability to become a remarkable luminescence sensor in chemical or biological applications.

\subsection{Detection of OTC in vitro and milk sample}

It is known that sensitivity of detection method strongly depends on the relevant experimental parameters. In this work, the OTC is determined based on ratiometric fluorescent strategy. Thus, it is much important to optimize the ratio of two fluorescent intensities. Both much weak and strong fluorescent intensity of SiNPs hampers its sensitivity toward OTC, as shown 
in Fig. S4a and b. $\dagger$ Herein, we use $4 \mathrm{mM}$ SiNPs as the optimum concentration to determine OTC (Fig. 3a). Besides, incubation time of analyte systems is also evaluated as shown in Fig. S5. $\dagger$ In the presence of OTC, the fluorescence of SiNPs changes quickly being finalized within 1 min and then kept constant with time. To obtain more reliable results, 2 min incubation after OTC addition is chosen before fluorescence measurement.

As a promising senor, the luminescent SiNPs are used to detect OTC in aqueous solution. Under the optimized conditions, the mixture combined with SiNPs and OTC represents double emission peaks at $436 \mathrm{~nm}$ and $502 \mathrm{~nm}$ when exited by $345 \mathrm{~nm}$. The fluorescence intensity of former at $436 \mathrm{~nm}$ is quenched, while the later one at $502 \mathrm{~nm}$ becomes stronger when the concentration of OTC is increased (Fig. 3a). Fig. 3b represents that a calibration curve for OTC detection with an excellent correlation $\left(I_{502} / I_{436}=0.064 C+0.389, R^{2}=0.9994\right)$ is achieved in the range of $0.2-20 \mu \mathrm{M}$, and the limit of detection (LOD) is as low as $0.18 \mu \mathrm{M}$ based on $\mathrm{S} / \mathrm{N}=3$.

Selectivity is another important parameter to evaluate the performance of a new fluorescent sensor. Hence, the interference from other TCs and biological ions $\left(\mathrm{Na}^{+}, \mathrm{K}^{+}, \mathrm{Cl}^{-}, \mathrm{CO}_{3}{ }^{2-}\right.$, $\mathrm{HCO}_{3}{ }^{-}, \mathrm{PO}_{4}{ }^{3-}, \mathrm{HPO}_{4}{ }^{2-}$ and $\mathrm{H}_{2} \mathrm{PO}_{4}{ }^{-}$) is investigated under the same conditions. As shown in Fig. 3c, it is observed that other TCs show almost no significant influence on the fluorescence intensity ratio of $I_{502} / I_{436}$, and the same result is obtained for all of the interfering biological ions (Fig. 3d). Therefore, these
Table 2 Determination results of OTC in milk samples

\begin{tabular}{lrllllll}
\hline & & \multicolumn{3}{l}{ Found $(\mu \mathrm{M})$} & & \\
Sample & $\begin{array}{l}\text { Spiked } \\
(\mu \mathrm{M})\end{array}$ & \multicolumn{1}{l}{1} & 2 & 3 & $\begin{array}{l}\text { Average } \\
\text { recovery }(\%)\end{array}$ & RSD $(\%)$ \\
\hline $1 \#$ & 1.00 & 0.91 & 1.13 & 1.07 & 1.04 & 0.11 \\
$2 \#$ & 5.00 & 5.03 & 4.86 & 5.28 & 1.01 & 0.21 \\
$3 \#$ & 10.00 & 10.16 & 9.69 & 9.82 & 0.99 & 0.24
\end{tabular}

findings reveal that the proposed method exhibits excellent selectivity toward OTC, especially among TCs' family. In comparison to other reported fluorescence methods for OTC, the present one has comparable performance, especially, in selectivity and fluorescence response strategy (Table S1 $\dagger$ ).

With respect to the practicality of the sensor to the real sample, the SiNPs are further tested in spiked milk samples with standard addition method and the results are listed in Table 2. It exhibits that recoveries of different known amounts of added OTC reached to $99 \%-104 \%$ in milk samples, and all the relative standard deviation (RSD) are smaller than $0.3 \%$. Thereby, the established radiometric fluorescence strategy using SiNPs is validated and successfully applied to detect OTC in real samples.

\subsection{Possible mechanism of fluorescence response}

To investigate the recognition mechanism of SiNPs toward OTC, SiNPs solution in absence and presence of OTC are firstly
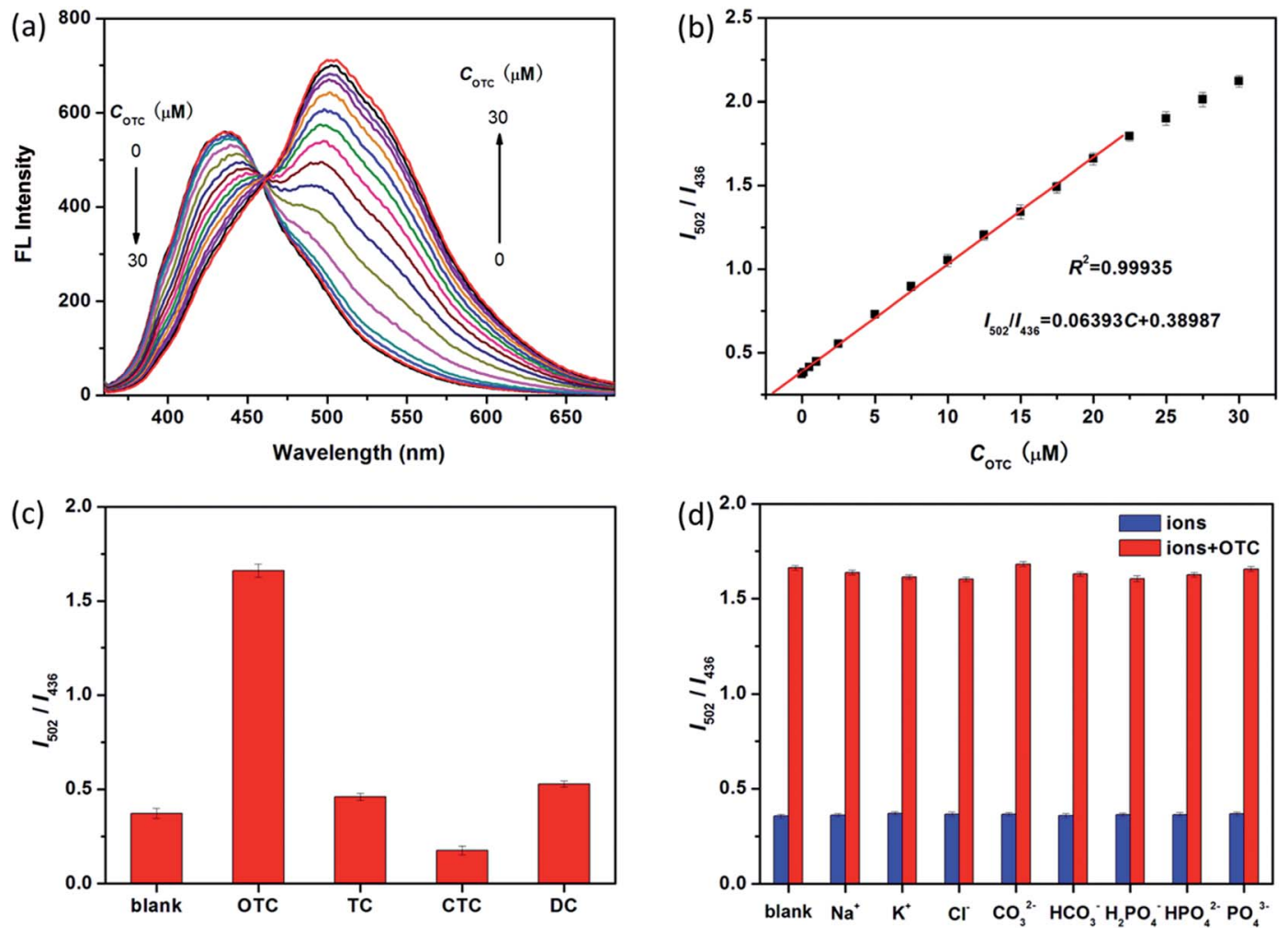

Fig. 3 (a) Fluorescence response of SiNPs (4 mM) toward different concentrations of OTC $(0,0.2,0.5,1.0,2.5,5.0,7.5,10.0,12.5,15.0,17.5,20.0$, 22.5, 25.0, 27.5 and $30.0 \mu \mathrm{M}$ ). (b) The linear relationship between the ratio of fluorescence intensities $\left(/ 502 / /_{436}\right)$ and OTC concentration exited by $345 \mathrm{~nm}$. (c) Comparison of $I_{502} / I_{436}$ of SiNPs and four SiNPs - TCs mixture $(4 \mathrm{mM}: 20 \mu \mathrm{M})$. (d) $I_{502} / I_{436}$ of SiNPs in absence and presence of various substances $(200 \mu \mathrm{M})$, in the presence (red) and absence (blue) of OTC $(20 \mu \mathrm{M})$, respectively. 
subjected to DLS and zeta potential measurements. As shown Fig. 4a, the size of SiNPs increases from $4.5 \mathrm{~nm}$ to $5.5 \mathrm{~nm}$, while the zeta potential changes from $2.58 \mathrm{mV}$ to $0.76 \mathrm{mV}$. According to the structural feature of OTC, it has the functional groups of hydroxyl, carbonyl and hydrogenated tetracene. The former two are potential binding sites in target the amino groups of SiNPs. Amounts of hydroxyl groups result in negative charging of OTC and attachment to electropositive SiNPs via electrostatic force. Hence, enlargement of grain size and decrease of zeta potential are both induced by the formation of SiNPs-OTC complex. In addition, Fig. S6† shows comparison of zeta potentials toward various TCs, such as OTC, TC, CTC and DC. It is clear to find that OTC possesses negative potential of $-9.96 \mathrm{mV}$; while only $-1.1,-3.69$ and $-1.02 \mathrm{mV}$ is obtained for CTC, TC and DC, respectively. Thus, the OTC can combine with positive charged SiNPs more easily than other TCs. That's one of the reasons why the luminescent SiNPs have good selectivity among TCs' family. In fact, it is also possible due to distinct structure and chemical properties of OTC, which will be further analyzed afterwards.

To further investigate the chemical bonds formed in SiNPsOTC complex, we evaluate and compare the FT-IR spectra of SiNPs, OTC and SiNPs-OTC complex. As shown in Fig. 4b, FT-IR spectrum of OTC shows a resonance at about $1630 \mathrm{~cm}^{-1}$, which is assigned to $\mathrm{C}=\mathrm{O}$ stretching vibration of carbonyl group. ${ }^{45}$ Upon complexed with SiNPs, the $\mathrm{C}=\mathrm{O}$ stretch of carbonyl group $\left(1630 \mathrm{~cm}^{-1}\right)$ diminished and a new sharp peak appears at
$1580 \mathrm{~cm}^{-1}$. Meanwhile, the absorption peak at $3440 \mathrm{~cm}^{-1}$ of $\mathrm{N}-\mathrm{H}$ bond bending vibration in SiNPs-OTC is almost vanished comparing to the pure SiNPs. Thus, the characteristic peak at $1580 \mathrm{~cm}^{-1}$ is not corresponding to $\mathrm{N}-\mathrm{H}$ stretching vibration of SiNPs but the $\mathrm{C}=\mathrm{N}$ bond from SiNPs-OTC complex, ${ }^{\mathbf{4 6 , 4 7}}$ via nucleophilic addition reaction between amino group in SiNPs and aldehyde group of OTC. This indicates the formation of $\mathrm{C}=\mathrm{N}$ contained Schiff base with fluorescence property when SiNPs solution is in presence of OTC. To confirm our assumption, a control experiment is employed, where OTC is added into APTES solution possessing amounts of amino groups (Fig. S7†). It is interesting to find that the mixture of OTC and APTES shows also a strong fluorescence emission at $505 \mathrm{~nm}$. Furthermore, FT-IR spectra displays characteristic peak at $1573 \mathrm{~cm}^{-1}$ assigned to $\mathrm{C}=\mathrm{N}$ bond in this mixture, suggesting APTES-OTC complex is formed with fluorescent Schiff base. Of note, a little difference indeed exists in fluorescence and FT-IR spectra toward SiNPs-OTC and APTES-OTC complex, due to the different origination. Besides, Fig. S8† indicates weight loss curves of SiNPs and SiNPs-OTC complex. In presence of OTC, the temperature of weight loss is shifted from high temperature side. This result suggests that the thermal stability of SiNPs is increased by the formation of covalent bonds $(\mathrm{C}=\mathrm{N})$ toward the surface of particles.

To investigate optical properties of SiNPs-OTC complex, the UV-vis absorption spectra of SiNPs, OTC and SiNPs-OTC
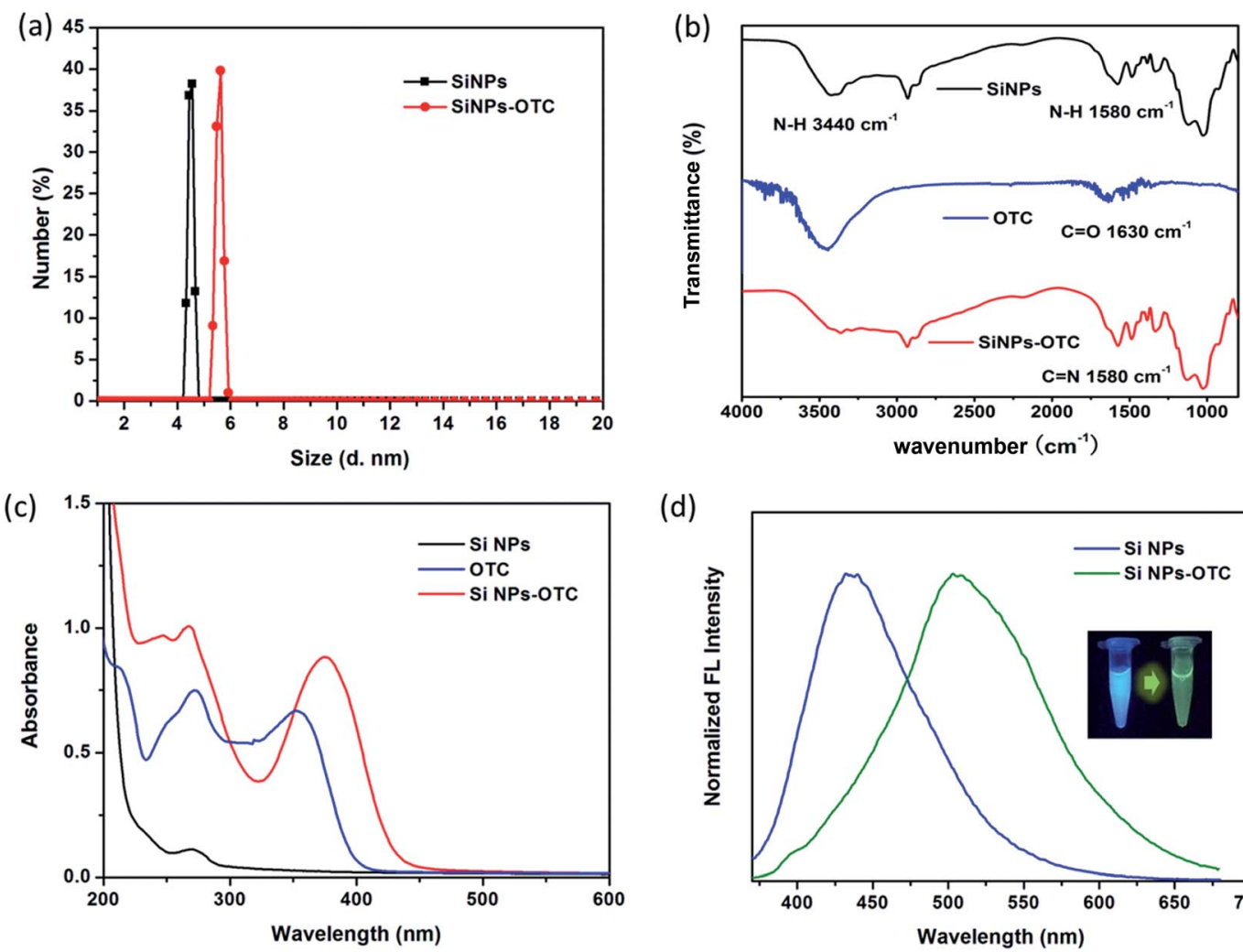

(d)

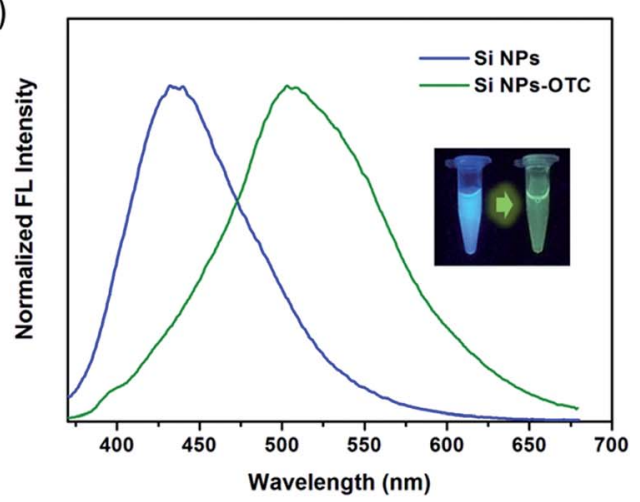

Fig. 4 (a) DLS size distribution of SiNPs and SiNPs-OTC complex through DLS measurements. (b) FT-IR and (c) absorption spectra of SiNPs (black), OTC (blue) and SiNPs-OTC complex (red). Fluorescence variation of SiNPs (blue) and SiNPs-OTC complex (green), inset is the corresponding photographs under UV lamp. 
complex are measured. As shown in Fig. 4c, a new intense absorption band at $375 \mathrm{~nm}$ assigned to Schiff base is observed in SiNPs-OTC complex, where it overlaps quite well with the excitation band of SiNPs centered at $345 \mathrm{~nm}$. Thereby, upon addition of OTC, the formed Schiff base in SiNPs-OTC complex absorbs the excitation spectrum toward SiNPs efficiently and the fluorescence intensity of SiNPs decreased based on the inner filter effect (IFE). Considering the IFE process between Schiff base and SiNPs in the complex, the SiNPs-OTC mixture can behave as "turn-off" fluorescence for SiNPs and "turn-on" fluorescence for Schiff base. Consequently, the fluorescence of the SiNPs changes from blue emission $(436 \mathrm{~nm})$ to green emission (502 nm) after titration of OTC (Fig. 4d). Table 1 shows the fluorescence decay profiles of SiNPs and SiNPs-OTC complex. The blue emission lifetimes of SiNPs are $7.71 \mathrm{~ns}$ and $9.63 \mu \mathrm{s}$, respectively, while the green emission lifetimes of SiNPs-OTC complex are $7.19 \mathrm{~ns}$ and $12.36 \mu \mathrm{s}$, demonstrating the changes of chemical environment around SiNPs and the formation of fluorescent Schiff base (Scheme 1).

\section{Conclusion}

In summary, amino groups terminated luminescent SiNPs have been designed for ratiometric visual detection of OTC in vitro and milk samples. Green emitting $\mathrm{C}=\mathrm{N}$ binding Schiff base is assembled onto the surface of blue emitting SiNPs, via nucleophilic addition reaction in the solution. The resulting SiNPsOTC mixture with a dual-emission property can prevent the fluorescence intensity from environment effects when using single luminescent sensor. Based on the inner filter effect, the blue emission at $436 \mathrm{~nm}$ quenches, while the green emission at $502 \mathrm{~nm}$ increase reversely when exited by $345 \mathrm{~nm}$. Consequently it brings the distinct changes of fluorescent color from blue to green. The intensity ratio of $I_{502} / I_{436}$ represents good linear relationship and relative low detection limit toward OTC. Furthermore, the strategy shows excellent selectivity particularly among TCs' family and satisfactory results in real sample tests.

\section{Conflicts of interest}

There are no conflicts to declare.

\section{Notes and references}

1 X. Pi, L. Zhang and D. Yang, J. Phys. Chem. C, 2012, 116, 21240-21243.

2 Y. Ding, R. Gresback, Q. Liu, S. Zhou, X. Pi and T. Nozaki, Nano Energy, 2014, 9, 25-31.

3 A. Politi, M. J. Cryan, J. G. Rarity, S. Yu and J. L. O'brien, Science, 2008, 320, 646-649.

4 Y. Su, X. Ji and Y. He, Adv. Mater., 2016, 28, 10567-10574.

5 R. K. Baldwin, K. A. Pettigrew, J. C. Garno, P. P. Power, G.-y. Liu and S. M. Kauzlarich, J. Am. Chem. Soc., 2002, 124, 1150-1151.
6 A. Shiohara, S. Hanada, S. Prabakar, K. Fujioka, T. H. Lim, K. Yamamoto, P. T. Northcote and R. D. Tilley, J. Am. Chem. Soc., 2010, 132, 248-253.

7 X. Li, Y. He, S. S. Talukdar and M. T. Swihart, Langmuir, 2003, 19, 8490-8496.

8 L. Mangolini and U. Kortshagen, Adv. Mater., 2007, 19, 25132519.

9 Z. Kang, Y. Liu, C. H. A. Tsang, D. D. D. Ma, X. Fan, N.-B. Wong and S.-T. Lee, Adv. Mater., 2009, 21, 661-664.

10 T. M. Atkins, A. Thibert, D. S. Larsen, S. Dey, N. D. Browning and S. M. Kauzlarich, J. Am. Chem. Soc., 2011, 133, 2066420667.

11 A. S. Heintz, M. J. Fink and B. S. Mitchell, Adv. Mater., 2007, 19, 3984-3988.

12 L. Zhu, X. Peng, H. Li, Y. Zhang and S. Yao, Sens. Actuators, B, 2017, 238, 196-203.

13 S. Chandra, G. Beaune, N. Shirahata and F. M. Winnik, J. Mater. Chem. B, 2017, 5, 1363-1370.

14 L. Meng, Y. Yuan, G. Pu and N. Xu, Anal. Methods, 2017, 9, 2553-2560.

15 Z. Jiannan, X. Chuan, F. Qiang, L. Ming, W. Baojun, F. Guodong, Y. Hongmei, H. Yanfu and S. Zhiguang, Nanotechnology, 2010, 21, 045501.

16 Y. Shi, Z. Chen, X. Cheng, Y. Pan, H. Zhang, Z. Zhang, C.-W. Li and C. Yi, Biosens. Bioelectron., 2014, 61, 397-403.

17 L. Ding, H. Liu, L. Zhang, L. Li and J. Yu, Sens. Actuators, B, 2018, 254, 370-376.

18 F. Gao, P. Cui, X. Chen, Q. Ye, M. Li and L. Wang, Analyst, 2011, 136, 3973-3980.

19 Z. Cao, F. Peng, Z. Hu, B. Chu, Y. Zhong, Y. Su, S. He and Y. He, Nanoscale, 2017, 9, 7602-7611.

20 K. Wang, X. He, X. Yang and H. Shi, Acc. Chem. Res., 2013, 46, 1367-1376.

21 F. Gao, X. Chen, Q. Ye, Z. Yao, X. Guo and L. Wang, Microchim. Acta, 2011, 172, 327-333.

22 R. S. Singer, R. Finch, H. C. Wegener, R. Bywater, J. Walters and M. Lipsitch, Lancet Infect. Dis., 2003, 3, 47-51.

23 A. Reilly and F. Käferstein, Aquacult. Res., 1997, 28, 735752.

24 A. L. Cinquina, F. Longo, G. Anastasi, L. Giannetti and R. Cozzani, J. Chromatogr. A, 2003, 987, 227-233.

25 W. John Blanchflower, R. J. McCracken, A. S. Haggan and D. Glenn Kennedy, J. Chromatogr. B, 1997, 692, 351-360.

26 V. Bersudsky, U. Rehany, Y. Tendler, E. Leffler, S. Selah and S. Rumelt, Graefe's Arch. Clin. Exp. Ophthalmol., 1999, 237, 617-620.

27 H. Wang, Y. Wang, S. Liu, J. Yu, Y. Guo, Y. Xu and J. Huang, Biosens. Bioelectron., 2016, 80, 471-476.

28 L. Meng, Q. Zhu, J.-H. Yin and N. Xu, J. Photochem. Photobiol., B, 2017, 173, 508-513.

29 N. Xu, H.-W. Li and Y. Wu, Anal. Chim. Acta, 2017, 958, 51-58.

30 X. An, S. Zhuo, P. Zhang and C. Zhu, RSC Adv., 2015, 5, 19853-19858.

31 F. Yuan, H. Zhao, Z. Zhang, L. Gao, J. Xu and X. Quan, RSC $A d v .$, 2015, 5, 58895-58901.

32 Z. Xu, X. Yi, Q. Wu, Y. Zhu, M. Ou and X. Xu, RSC Adv., 2016, 6, 89288-89297. 
33 S. Xu, X. Li, Y. Mao, T. Gao, X. Feng and X. Luo, Anal. Bioanal. Chem., 2016, 408, 2955-2962.

34 H. Zhang, C. Fang, S. Wu, N. Duan and Z. Wang, Anal. Biochem., 2015, 489, 44-49.

35 B. Ghosh, Y. Masuda, Y. Wakayama, Y. Imanaka, J.-i. Inoue, K. Hashi, K. Deguchi, H. Yamada, Y. Sakka, S. Ohki, T. Shimizu and N. Shirahata, Adv. Funct. Mater., 2014, 24, 7151-7160.

36 Q. Zhao, R. Zhang, D. Ye, S. Zhang, H. Chen and J. Kong, ACS Appl. Mater. Interfaces, 2017, 9, 2052-2058.

37 Y. Feng, Y. Liu, C. Su, X. Ji and Z. He, Sens. Actuators, B, 2014, 203, 795-801.

38 N. H. N. Kamarudin, A. A. Jalil, S. Triwahyono, N. F. M. Salleh, A. H. Karim, R. R. Mukti, B. H. Hameed and A. Ahmad, Microporous Mesoporous Mater., 2013, 180, 235-241.

39 V. R. Rai and S. Agarwal, Chem. Mater., 2011, 23, 2312-2316.
40 R. Tohmon, H. Mizuno, Y. Ohki, K. Sasagane, K. Nagasawa and Y. Hama, Phys. Rev. B, 1989, 39, 1337-1345.

41 Y. D. Glinka, S. H. Lin and Y. T. Chen, Phy. Rev. B, 2000, 62, 4733-4743.

42 Y. Yang, J. Cui, M. Zheng, C. Hu, S. Tan, Y. Xiao, Q. Yang and Y. Liu, Chem. Commun., 2012, 48, 380-382.

43 Q. Li, T.-Y. Luo, M. Zhou, H. Abroshan, J. Huang, H. J. Kim, N. L. Rosi, Z. Shao and R. Jin, ACS Nano, 2016, 10, 8385-8393.

44 S. Chandra, B. Ghosh, G. Beaune, U. Nagarajan, T. Yasui, J. Nakamura, T. Tsuruoka, Y. Baba, N. Shirahata and F. M. Winnik, Nanoscale, 2016, 8, 9009-9019.

45 Z. Li, S. Guo, Z. Yuan and C. Lu, Sens. Actuators, B, 2017, 241, 821-827.

46 X. Qu, A. Wirsén and A. C. Albertsson, Polymer, 2000, 41, 4589-4598.

47 A. Singh, S. Narvi, P. Dutta and N. Pandey, Bull. Mater. Sci., 2006, 29, 233-238. 